\title{
Effect of Surface Roughness on MHD Couple Stress Squeeze-Film Characteristics between a Sphere and a Porous Plane Surface
}

\author{
M. Rajashekar' ${ }^{1}$ and Biradar Kashinath ${ }^{2}$ \\ ${ }^{1}$ Department of Mathematics, Govt. PU. College for Girls, Raichur 584 101, India \\ ${ }^{2}$ Department of Mathematics, Govt. First Grade College, Shorapur, Gulbarga 585 224, India
}

Correspondence should be addressed to M. Rajashekar, srajashekarm@gmail.com

Received 1 August 2012; Revised 7 November 2012; Accepted 8 November 2012

Academic Editor: N. M. Bujurke

Copyright (C) 2012 M. Rajashekar and B. Kashinath. This is an open access article distributed under the Creative Commons Attribution License, which permits unrestricted use, distribution, and reproduction in any medium, provided the original work is properly cited.

\begin{abstract}
The combined effects of couple stress and surface roughness on the MHD squeeze-film lubrication between a sphere and a porous plane surface are analyzed, based upon the thin-film magnetohydrodynamic (MHD) theory. Using Stoke's theory to account for the couple stresses due to the microstructure additives and the Christensen's stochastic method developed for hydrodynamic lubrication of rough surfaces derives the stochastic MHD Reynolds-type equation. The expressions for the mean MHD squeezefilm pressure, mean load-carrying capacity, and mean squeeze-film time are obtained. The results indicate that the couple stress fluid in the film region enhances the mean MHD squeeze-film pressure, load-carrying capacity, and squeeze-film time. The effect of roughness parameter is to increase (decrease) the load-carrying capacity and lengthen the response time for azimuthal (radial) roughness patterns as compared to the smooth case. Also, the effect of porous parameter is to decrease the load-carrying capacity and increase the squeeze-film time as compared to the solid case.
\end{abstract}

\section{Introduction}

With the development of modern machine equipments, the increasing use of fluids containing microstructures such as additives, suspensions, and long-chained polymers has received great attention. Newtonian fluid approximation (which neglects the size of fluid particles) is not a satisfactory engineering approach for the study fluids with microstructure additives. Since, the flow behaviours of non-Newtonian fluids cannot be described accurately by the classical Continuum theory, many microcontinuum theories have been proposed [1-3]. Among them, the Stoke's [3] microcontinuum theory is the simplest generalization of the classical Newtonian theory of fluids that allows for polar effects such as the presence of couple stresses and body couples. This couple stress fluid model is intended to account for the particle-size effects and is important for applications of pumping fluids such as animal bloods, liquid crystals, polymer thickened oils, and complex fluids. Ramanaiah [4] analyzed squeeze films between finite plates lubricated by fluids with couple stress. Several researchers have applied the Stoke's [3] microcontinuum theory to investigate the effect of couple stresses on the performance of different types of fluid-film bearings.

The use of liquid metals has recently become of interest due to thin highly conducting properties. There is a possibility of increasing the load-carrying capacity by using lubricants in the presence of an applied magnetic field. The effect of surface roughness plays an important role in engineering science and industrial applications. In bearings, surface roughness is a measure of the texture of a surface. It is quantified by the vertical deviations of a real surface from its ideal form. If these deviations are large, the surface is rough; if they are small, the surface is smooth. Rough surfaces usually wear more quickly and have higher friction coefficients than smooth surfaces. Roughness is often a good predictor of the performance of a mechanical component, since irregularities in the surface may form nucleation sites for cracks or corrosion. In bearings, the height of the roughness asperities and the mean separation of the sliding surfaces are of the same order. Thus, it appeared normal to 
view the film thickness in a bearing as a stochastic process characterized by a number of statistical parameters.

Traditionally, the analysis of porous squeeze-film bearings was based on the Darcy's model, where the fluid flow in the porous matrix obeys Darcy's law and at the bearing/ film interface the no-slip condition was assumed. The term squeeze film applies to the case of two approaching surfaces which attempt to displace a viscous fluid between them. For very thin film, viscous forces offer a high resistance to such fluid motion, which, in turn, tends to inhibit the approach of the bounding surfaces. If one or both of the approaching surfaces are porous, the lubricant not only gets squeezed out from the sides but also bleeds into the pores of the porous matrix, thus reducing the time of approach of the surfaces considerably. Despite this advantage, porous bearings have proved to be useful because of their self-lubricating characteristics, low initial cost, and design simplicity.

In recent years, the magnetohydrodynamic lubrication phenomenon has many industrial applications, because of increased use of liquid metal lubricants in high temperature. A number of theoretical and experimental investigations have been made on the effects of magnetohydrodynamic lubrication $[5,6]$. There is a possibility of increasing the load-carrying capacity by using lubricants in the presence of an applied magnetic field. Further, the above result has been used in modifying the squeeze-film action of bearing of an externally applied magnetic field. The squeeze film characteristics between a sphere and a flat plate was analyzed by Gould [7] and Conway and Lee [8]. Hamza [9] studied the motion of an electrically conducting fluid film squeezed between two parallel disks in the presence of a magnetic field and found that, by applying a magnetic field the load will increase and correspondingly improvement in the lubrication characteristics of liquid-metal lubricants. Lin [10] analyzed the couple stress squeeze-film characteristics between a sphere and a flat plate. It is well established that when an electrically conducting fluid flows under the influence of electromagnetic fields, the pressure gradient is increased considerably due to the body forces [11]. The interaction of the flow of the fluids through permeable media with magnetic field has received special attention [12]. The squeeze film between porous plates was studied by Verma [13] and Bhat and Daheri [14] and showed that the application of a magnetic fluid lubricant improved the performance of the squeeze film. The problem of squeeze film porous metal bearings has been studied by Prakash and Vij [15]. Shah and Bhat [16] analyzed the effects of magnetic fluid lubricant on the squeeze film between curved porous rotating circular plates in the presence of an external magnetic field applied to the lower plate.

Many workers have made investigations on the hydrodynamic lubrication of rough surfaces using stochastic approaches. Christensen [17] and Elord [18] developed the stochastic models for hydrodynamic lubrication of rough surfaces and also derived the generalized form of the Reynold's equation applicable to rough bearings. A stochastic model to study the effect of surface roughness in porous bearings was developed by Prakash and Tiwari
[19] on the basis of Christensen's stochastic theory. Using this theory, Prakash and Tiwari [20] and Gururajan and Prakash [21] analyzed various types of porous bearings with Newtonian lubricants. Naduvinamani et al. [22] have used this model to study the combined effects of surface roughness and lubricant additives oil various porous bearing systems and found that the presence of lubricant additives has a considerable influence on the characteristics of rough porous bearings. Using the same model, the effect of surface roughness on MHD lubrication flow between rectangular plates was analyzed by Bujurke and Kudenatti [23] and they concluded that by the proper choice of the lubricants with additives on the bearing surface improves its performance. The MHD squeeze-film characteristics between a sphere and a plane surface was studied by Chou et al. [24]. Recently, Naduvinamani and Rajashekar [25] studied the MHD couple stress squeeze-film characteristics between a sphere and a plane surface. They found that the MHD squeeze-film pressure, load-carrying capacity, increases in the presence of externally applied magnetic field and for large values of Hartmann number, the response time also increases. In the current study, we analyze the effects of couple stress and surface roughness on the magnetohydrodynamic squeeze-film characteristics between a sphere and a porous plane surface, which have not been studied so far. Hence, in this paper an attempt has been made to analyze the combined effects of couple stress and surface roughness on the MHD squeeze-film characteristics between a sphere and a porous plane surface. Expressions for the MHD squeezefilm pressure and load carrying capacity are obtained. Results are compared with the corresponding smooth and solid case studied by Naduvinamani and Rajashekar [25], Newtonian case by Chou et al. [24], and nonmagnetic Newtonian lubricant case $[7,8]$.

\section{Mathematical Formulation of the Problem}

The physical configuration of a rough porous squeeze-film bearing is shown in Figure 1. A solid sphere of radius $R$ is approaching the rough porous bearing of wall thickness $H_{0}$ with a velocity $(\partial h / \partial t)$ under a constant load. An incompressible electrically conducting fluid is considered as a lubricant in the film and also in the porous region between two surfaces. An externally uniform transverse magnetic field $B_{0}$ is applied to the lower surface.

The stochastic film thickness $H$ is represented by

$$
H=h+h_{s}(r, \theta, \xi) \text {, }
$$

where $h=h_{m}+r^{2} / 2 R$ (provided $R \gg r$ ) denotes the nominal smooth part of the film geometry, while $h_{s}$ is the part due to the surface asperities measured from the nominal level and is a randomly varying quantity of zero mean and $\xi$ is in index describing the definite roughness arrangement, hence for a given value of $\xi$, the surface roughness component $h_{s}$ of the film thickness becomes a deterministic function of the space variables.

Under the usual assumption of hydrodynamic lubrication theory applicable to thin-film, the continuity equation 


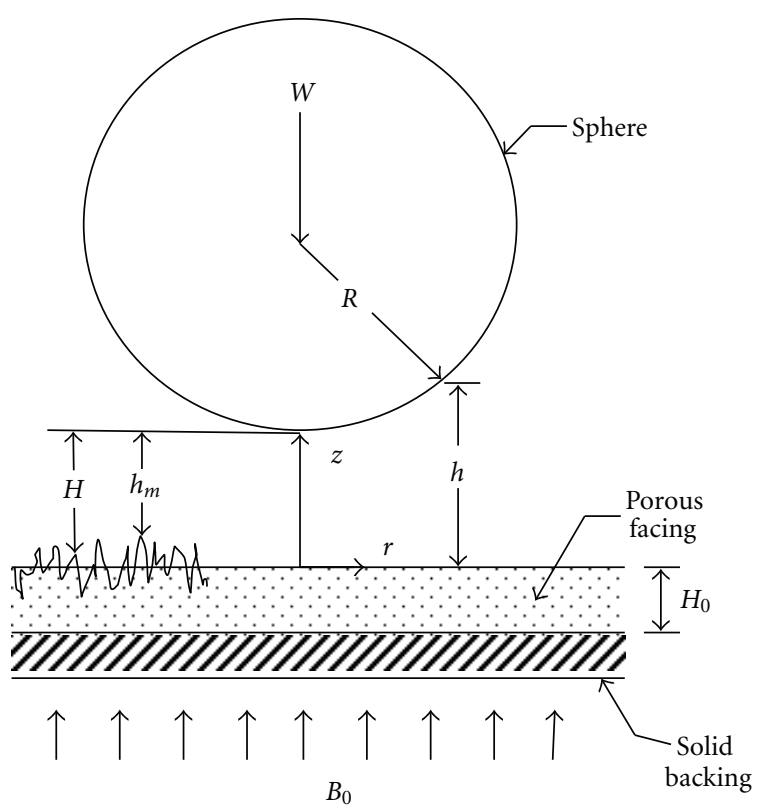

FIGURE 1: Squeeze-film geometry between a sphere and a porous plane surface with roughness in the presence of a transverse magnetic field.

and the magnetohydrodynamic (MHD) momentum equations in polar coordinates $(r, \theta, z)$ becomes

$$
\begin{gathered}
\mu \frac{\partial^{2} u}{\partial z^{2}}-\eta \frac{\partial^{4} u}{\partial z^{4}}-\sigma B_{0}^{2} u=\frac{\partial p}{\partial r} \\
\frac{\partial p}{\partial z}=0 \\
\frac{1}{r} \frac{\partial}{\partial r}(r u)+\frac{\partial w}{\partial z}=0
\end{gathered}
$$

The flow of conducting lubricant in the porous region is governed by the modified Darcy's law [14]

$$
\begin{gathered}
u^{*}=-\frac{k}{\mu} \frac{\partial p^{*}}{\partial r} \frac{1}{c^{\prime 2}}, \\
w^{*}=-\frac{k}{\mu(1-\beta)} \frac{\partial p^{*}}{\partial z}, \\
\frac{1}{r} \frac{\partial}{\partial r}\left(r u^{*}\right)+\frac{\partial w^{*}}{\partial z}=0,
\end{gathered}
$$

where $p^{*}$ is the pressure in the porous matrix and $c^{\prime}=$ $\left(1-\beta+(k / m)\left(M^{2} / h_{m_{0}}^{2}\right)\right)^{1 / 2}$.

The relevant boundary conditions for the velocity components are

$$
\begin{gathered}
u=0, \quad w=0, \quad \text { at } z=0, \\
\frac{\partial^{2} u}{\partial z^{2}}=0 \quad \text { at } z=0, \\
u=0, \quad w=\frac{\partial H}{\partial t}, \quad \text { at } z=H, \\
\frac{\partial^{2} u}{\partial z^{2}}=0 \quad \text { at } z=H .
\end{gathered}
$$

The boundary conditions given in (7) and (9) are due to vanishing of couple stresses on the solid boundaries at $z=0$ and $z=H$, respectively.

The radial velocity component $u$ is obtained by solving (2) with the boundary conditions (6)-(9) in the form:

$$
\begin{aligned}
u=\frac{h_{m_{0}}^{2}}{\mu M^{2}} \frac{\partial p}{\partial r}[\{ & -\left(\frac{B^{2}}{A^{2}-B^{2}}\right)\left(\frac{\cosh (A / 2 l)(2 z-H)}{\cosh (A H / 2 l)}\right) \\
& \left.\left.+\left(\frac{A^{2}}{A^{2}-B^{2}}\right)\left(\frac{\cosh (B / 2 l)(2 z-H)}{\cosh B H / 2 l}\right)\right\}-1\right],
\end{aligned}
$$

where $h_{m_{0}}$ denotes the initial minimum film thickness and the magnetization Hartmann number $M$ defined by

$$
M=B_{0} h_{m_{0}}\left(\frac{\sigma}{\mu}\right)^{1 / 2}
$$

and the couple stress parameter

$$
l=\left(\frac{\eta}{\mu}\right)^{1 / 2}
$$

where the dimension of $l$ is of length and is identified as the molecular length of the polar suspensions in a nonpolar fluid.

\section{Solution of the Problem}

The non-Newtonian MHD couple stress Reynolds-type equation for the squeeze-film pressure can be obtained by substituting for $u$ from (10) into the continuity (4) and integrating across the film thickness $z$ and also using the 
boundary conditions of $w$ given in (6) and (9), in the following form:

$$
\frac{1}{r} \frac{\partial}{\partial r}\left[\left\{\frac{k H_{0}(1-\beta)}{c^{\prime 2}}+\frac{h_{m_{0}}^{2}}{M^{2}} F(H, l, M)\right\}\left(r \frac{\partial p}{\partial r}\right)\right]=\mu \frac{\partial H}{\partial t},
$$

where

$$
\begin{aligned}
F(H, l, M)=\frac{2 l}{A^{2}-B^{2}} & {\left[\left(\frac{B^{2}}{A}\right) \tanh \left(\frac{A H}{2 l}\right)\right.} \\
& \left.-\left(\frac{A^{2}}{B}\right) \tanh \left(\frac{B H}{2 l}\right)\right]+H .
\end{aligned}
$$

Let $f\left(h_{s}\right)$ be the probability density function of the stochastic film thickness $h_{s}$.

Taking the stochastic average of (13) with respect to $f\left(h_{s}\right)$, we obtain

$$
\begin{gathered}
\frac{1}{r} \frac{\partial}{\partial r}\left[\left\{\frac{k H_{0}(1-\beta)}{c^{\prime 2}}+\frac{h_{m_{0}}^{2}}{M^{2}} E(F(H, l, M))\right\}\left(r \frac{\partial E(p)}{\partial r}\right)\right] \\
=\mu \frac{\partial E(H)}{\partial t} \\
E(\cdot)=\int_{-\infty}^{\infty}(\cdot) f\left(h_{s}\right) d h_{s} .
\end{gathered}
$$

In accordance with Christensen [17], we assume that

$$
f\left(h_{s}\right)= \begin{cases}\frac{35}{32 c^{7}}\left(c^{2}-h_{s}^{2}\right)^{3}, & c<h_{s}<c \\ 0, & \text { elsewhere }\end{cases}
$$

where $\sigma^{\prime}=c / 3$ is the standard deviation.

Introducing the nondimensional quantities

$$
\begin{gathered}
\bar{r}=\frac{r}{R}, \quad \bar{h}_{m_{0}}=\frac{h_{m}}{h_{m_{0}}}, \quad \beta=\frac{h_{m_{0}}}{R}, \\
\bar{H}=\frac{H}{h_{m_{0}}}=\bar{h}+\bar{h}_{s}, \quad \bar{h}_{s}=\frac{h_{s}}{h_{m_{0}}}, \\
\bar{h}=\frac{h}{h_{m_{0}}}=\bar{h}_{m}+\frac{\bar{r}^{2}}{2 \beta}, \quad \bar{p}=\frac{E(p) h_{m_{0}}^{2}}{\mu R\left(-d h_{m} / d t\right)}
\end{gathered}
$$

into (15) where $\bar{h}_{m_{0}}$ denotes the nondimensional minimum film height.

The nondimensional non-Newtonian MHD couple stress Reynolds-type equation is obtained in the form:

$$
\frac{\partial}{\partial \bar{r}}\left[\left\{\frac{\psi(1-\beta)}{c^{2}}+\frac{E(F(\bar{H}, \bar{l}, M))}{M^{2}}\right\}\left(\bar{r} \frac{\partial E(\bar{p})}{\partial \bar{r}}\right)\right]=-\frac{\bar{r}}{\beta},
$$

where

$$
\begin{array}{r}
F(\bar{H}, \bar{l}, M)=\frac{\bar{l}^{2}}{\bar{A}^{2}-\bar{B}^{2}}\left[\left(\frac{\bar{B}^{2}}{\bar{A}}\right) \tanh \left(\frac{\overline{A H}}{\bar{l}}\right)\right. \\
-\left(\frac{\bar{A}^{2}}{\bar{B}}\right) \tanh \left(\frac{\overline{B H}}{\bar{l}}\right) \\
\bar{l}=\frac{2 l}{h_{m_{0}}}, \quad \bar{A}^{2}=\frac{1+\sqrt{1-\bar{l}^{2} M^{2}}}{2}, \\
\bar{c}=\frac{c}{h_{m_{0}}} \quad \text { (the roughness parameter), } \\
\psi=\frac{k H}{h_{m_{0}}^{3}} \quad \text { (the porous parameter). }
\end{array}
$$$$
\left.-\left(\frac{\bar{A}^{2}}{\bar{B}}\right) \tanh \left(\frac{\overline{B H}}{\bar{l}}\right)\right]+\bar{H},
$$

In accordance with the Christensen [17] stochastic theory, the analysis is done for the two types of one-dimensional surface roughness patterns, namely one-dimensional radial roughness pattern and one-dimensional azimuthal roughness pattern.

For the one-dimensional radial roughness pattern, the roughness striations are in the form of ridges and valleys in the $r$-direction in this case the nondimensional film thickness assumes the form:

$$
\bar{H}=\bar{h}+\bar{h}_{s}(\theta, \xi)
$$

For the one-dimensional radial roughness pattern, the roughness striations are in the form of ridges and valleys in the $\theta$-direction in this case the nondimensional film thickness assumes the form:

$$
\bar{H}=\bar{h}+\bar{h}_{s}(r, \xi)
$$

Then the modified-stochastic Reynold's type (19) for these two types of roughness pattern take the form:

$$
\frac{\partial}{\partial \bar{r}}\left[G(\bar{H}, \bar{l}, M, \bar{c}, \psi)\left(\bar{r} \frac{\partial E(\bar{p})}{\partial \bar{r}}\right)\right]=-\frac{\bar{r}}{\beta},
$$

where

$$
G(\bar{H}, \bar{l}, M, \bar{c}, \psi)=\left\{\begin{array}{l}
{\left[\frac{E(F(\bar{H}, \bar{l}, M))}{M^{2}}+\frac{\psi(1-\beta)}{c^{2}}\right],} \\
\text { for radial roughness } \\
{\left[\frac{M^{2}}{E(F(\bar{H}, \bar{l}, M))}+\frac{\psi(1-\beta)}{c^{2}}\right],} \\
\text { for azimuthal roughness }
\end{array}\right.
$$




$$
\begin{gathered}
E(F(\bar{H}, \bar{l}, M)) \\
=\frac{\bar{l}^{2}}{\bar{A}^{2}-\bar{B}^{2}}\left[\left(\frac{\bar{B}^{2}}{\bar{A}}\right) \tanh \left(\frac{\overline{A H}}{\bar{l}}\right)\right. \\
\left.-\left(\frac{\bar{A}^{2}}{\bar{B}}\right) \tanh \left(\frac{\overline{B H}}{\bar{l}}\right)\right]+E(\bar{H}) \\
E\left(\tanh \left(\frac{\overline{A H}}{\bar{l}}\right)\right)=\frac{35}{32 \bar{c}^{7}} \int_{-\bar{c}}^{\bar{c}}\left(\bar{c}^{2}-\bar{h}_{s}^{2}\right)^{3} \tanh \left(\frac{\overline{A H}}{\bar{l}}\right) d \bar{h}_{s}, \\
E\left[\frac{1}{F}(\bar{H}, \bar{l}, M)\right]=\frac{35}{32 \bar{c}^{7}} \int_{-\bar{c}}^{\bar{c}} \frac{\left(\bar{c}^{2}-\bar{h}_{s}^{2}\right)^{3}}{F(\bar{H}, \bar{l}, M)} d \bar{h}_{s} .
\end{gathered}
$$

The relevant boundary conditions for the mean squeeze-film pressure field are

$$
\begin{array}{cl}
\bar{p}=0 & \text { at } \bar{r}=1, \\
\frac{d \bar{p}}{d \bar{r}}=0 & \text { at } \bar{r}=0 .
\end{array}
$$

Integrating the nondimensional stochastic Reynold's type Equation (23) and using boundary conditions (25a) and (25b),

$$
\bar{p}=\frac{1}{2 \beta} \int_{\bar{r}=\bar{r}}^{\bar{r}=1}\left\{\frac{\bar{r}}{G(\bar{H}, \bar{l}, M, \bar{c}, \psi)}\right\} d \bar{r}
$$

which is nondimensional MHD mean squeeze-film pressure.

The nondimensional MHD mean load-carrying capacity is derived by integrating the nondimensional MHD mean squeeze-film pressure acting on the sphere as

$$
\begin{aligned}
\bar{W} & =\frac{E(W) h_{m_{0}}^{2}}{\mu R^{3}\left(-d h_{m} / d t\right)} \\
& =\frac{\pi}{\beta} \int_{\bar{r}=0}^{1}\left\{\int_{\bar{r}=\bar{r}}^{\bar{r}=1} \frac{\bar{r}}{G(\bar{H}, \bar{l}, M, \bar{c}, \psi)} d \bar{r}\right\} \bar{r} d \bar{r} .
\end{aligned}
$$

For a steady applied load $E(W)$, we introduce the following nondimensional response time

$$
\bar{t}=\frac{E(W) h_{m_{0}}^{2}}{\mu R^{4}} t
$$

The time-height relationship can be obtained from (27)

$$
\frac{d \bar{h}_{m}}{d \bar{t}}=-\frac{1}{\pi \int_{\bar{r}=0}^{1}\left\{\int_{\bar{r}=\bar{r}}^{\bar{r}=1}(\bar{r} / G(\bar{H}, \bar{l}, M, \bar{c}, \psi)) d \bar{r}\right\} \bar{r} d \bar{r}} .
$$

Using the fourth-order Runge-Kutta method, the minimum film height at the central position can be numerically evaluated with the initial condition $\bar{h}_{m}=1$ at $\bar{t}=0$.

Following are the limiting cases of the present study.

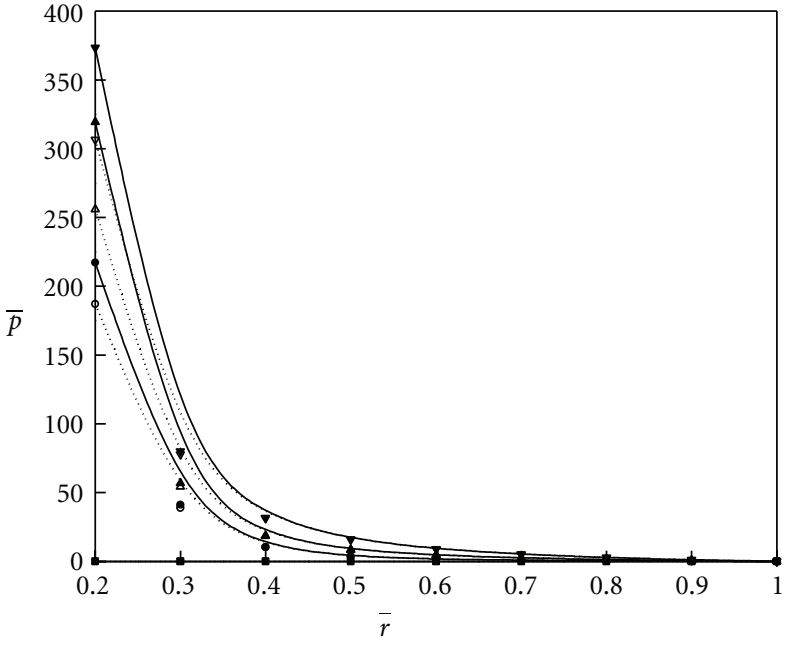

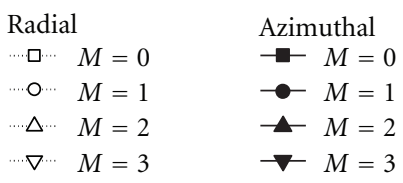

FIGURE 2: Variation of nondimensional mean film pressure $\bar{p}$ with $\bar{r}$ for different values of $M$ with $\beta=0.05, \bar{l}=0.3, \bar{c}=0.3$, and $\psi=0.01$.

(1) When $\bar{c} \rightarrow 0$ and $\psi \rightarrow 0$, the function $G(\bar{H}, \bar{l}$, $M, \bar{c}, \psi) \rightarrow F(\bar{h}, \bar{l}, M)$ and the results of Naduvinamani and Rajashekar [25] can be recovered from (27) and (29).

(2) When $\bar{l} \rightarrow 0, \bar{c} \rightarrow 0$ and $\psi \rightarrow 0$ the function $G(\bar{H}$, $M, \bar{c}, \psi) \rightarrow F(\bar{h}, M)$ and the results of Chou et al. [24] can be recovered from (27) and (29).

(3) As $M \rightarrow 0, \bar{l} \rightarrow 0, \bar{c} \rightarrow 0$ and $\psi \rightarrow 0$, the modified Reynold's type equation (29) reduces to the nonconducting smooth Newtonian lubricant case studied by Gould [7] and Conway and Lee [8] on squeeze-film characteristics between a sphere and a plane surface.

\section{Results and Discussion}

The combined effects of couple stress and surface roughness on the MHD squeeze-film characteristics of a sphere and a porous plane surface lubricated with an electrically conducting fluid in the presence of a transverse magnetic field are analyzed in this study. The Hartmann number, $M$, signifies the effects of externally applied magnetic field, the couple stress parameter, $\bar{l}$, signifies the effects of couple stresses, the porous parameter, $\psi$, signifies the effect of permeability, and the roughness parameter, $\bar{c}$, signifies the effect of surface roughness upon the MHD squeeze-film characteristics.

4.1. MHD Squeeze-Film Pressure. Figure 2 shows the variation of nondimensional mean pressure $\bar{p}$ with the axial coordinate $\bar{r}$ as a function of magnetization number $M$ with 

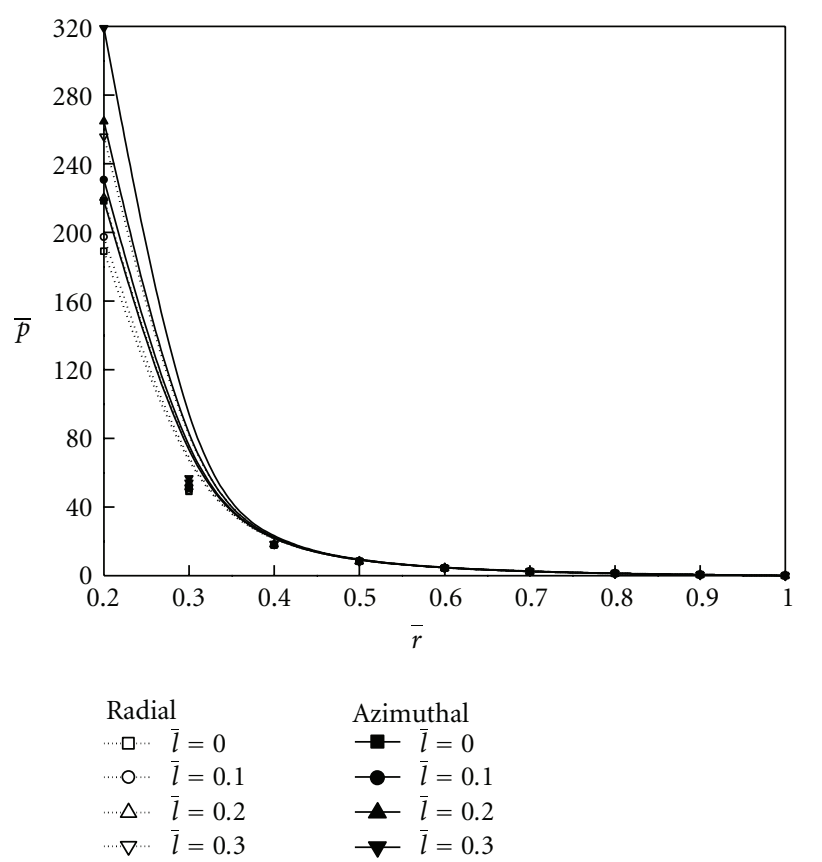

FIGURE 3: Variation of nondimensional mean film pressure $\bar{p}$ with $\bar{r}$ for different values of $\bar{l}$ with $\beta=0.05, M=2, \bar{c}=0.3$, and $\psi=0.01$.

the parametric values $\beta=0.05, \bar{h}_{m}=0.3, \bar{l}=0.3, \bar{c}=$ 0.3 , and $\psi=0.01$ for both types of roughness patterns. It is observed that the effect of magnetic field is to increase $\bar{p}$ in either cases as compared to the magnetic case. At $\bar{r}=0$ (i.e., in the neighbourhood of the position of minimum film height), due to the effects of magnetic field, the higher squeeze-film pressure is observed. Further, the increase in $\bar{p}$ is more pronounced for the azimuthal roughness pattern as compared to the radial roughness pattern.

The variation of $\bar{p}$ with $\bar{r}$ as a function of the couple stress parameter $\bar{l}$ with $\beta=0.05, \bar{h}_{m}=0.3, M=2, \bar{c}=$ 0.3 , and $\psi=0.01$ for both types of roughness patterns is shown in Figure 3. It is found that due to the presence of couple stress, $\bar{p}$ increases/decreases for azimuthal/radial roughness pattern.

Figure 4 displays the variation of $\bar{p}$ with $\bar{r}$ as a function of the roughness parameter $\bar{c}$ with $\beta=0.05, \bar{h}_{m}=0.3, M=$ $2, \bar{l}=0.3$, and $\psi=0.01$ for both types of roughness patterns. It is observed that, $\bar{p}$ increases/decreases for azimuthal/radial roughness pattern. As the vertical deviations of the surface increase, the squeeze-film pressure also increases significantly.

The variation of $\bar{p}$ with $\bar{r}$ as a function of porous parameter $\psi$ with $\beta=0.05, \bar{h}_{m}=0.3, M=2, \bar{l}=0.3$, and $\bar{c}=0.3$ for both types of roughness patterns is presented in Figure 5. It is found that the effect of permeability is to decrease $\bar{p}$ for increasing values of $\psi$ for both types of roughness patterns. As the sphere moves downwards, the fluid is forced into the pores of the porous material and hence resulting in the lapse of pressure on the fluid in the film region can be observed.
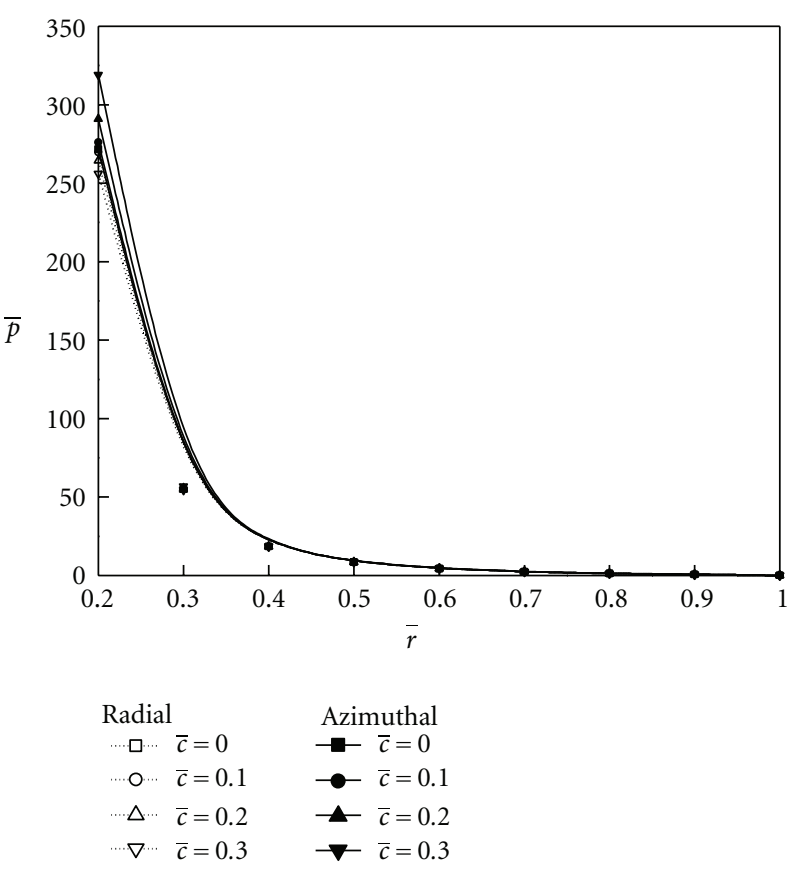

FIGURE 4: Variation of nondimensional mean film pressure $\bar{p}$ with $\bar{r}$ for different values of $\bar{c}$ with $\beta=0.05, M=2, \bar{l}=0.3$, and $\psi=0.01$.

4.2. MHD Load-Carrying Capacity. Figure 6 shows the variation of nondimensional mean load-carrying capacity $\bar{W}$ with magnetization number $M$ as a function of $\bar{h}_{m}$ with $\beta=0.05, \bar{l}=0.3, \bar{c}=0.3$, and $\psi=0.01$ for both types of roughness patterns. It is interesting to note that the effect of magnetic field is to increase $\bar{W}$ in either cases as compared to nonmagnetic case. Further, the increase in $\bar{W}$ is more pronounced for azimuthal roughness pattern as compared to the radial roughness pattern.

The variation of nondimensional mean load-carrying capacity $\bar{W}$ with magnetization number $M$ as a function of couple stress parameter, $\bar{l}$, with $\beta=0.05, \bar{c}=0.3$, and $\psi=$ 0.01 for both types of roughness patterns is plotted in Figure 7. It is observed that the effect of couple stresses enhances $\bar{W}$ in either cases as compared to Newtonian case.

Figure 8 shows the variation of $\bar{W}$ with $M$ as a function of roughness parameter $\bar{c}$ with $\beta=0.05, \bar{l}=0.3$, and $\psi=$ 0.01 for both types of roughness patterns. It is observed that the effect of azimuthal/radial roughness patterns is to increase/decrease $\bar{W}$ as compared to the corresponding smooth case (i.e., $\bar{c}=0$ ). At $\bar{c}=0.3$ (i.e., when the vertical deviations are large) it is found that, the increase/decrease in $\bar{W}$ is more pronounced for the azimuthal/radial roughness pattern. The large amount of load is delivered for azimuthal roughness pattern as compared to the radial roughness pattern.

The variation of $\bar{W}$ with $M$ for different values of $\psi$ is presented in Figure 9. It is observed that the effect of permeability parameter $\psi$ is to decrease the mean loadcarrying capacity as compared to the corresponding solid case $(\psi=0)$ for both types of roughness patterns. The 


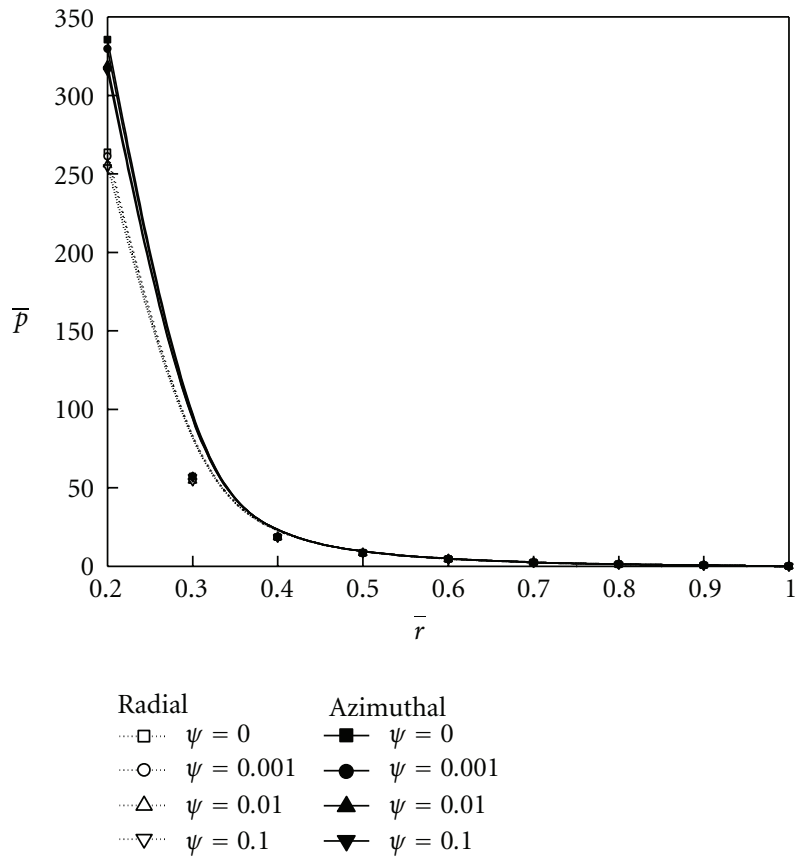

FIGURE 5: Variation of nondimensional mean film pressure $\bar{p}$ with $\bar{r}$ for different values of $\psi$ with $\beta=0.05, M=2, \bar{l}=0.3$, and $\bar{c}=0.3$.

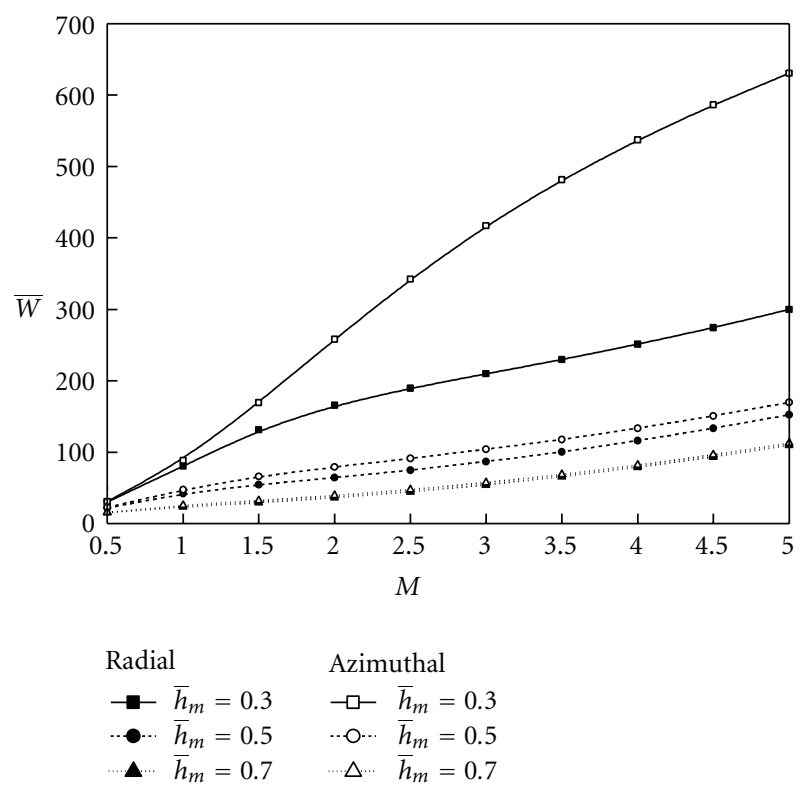

FIGURE 6: Variation of nondimensional mean load-carrying capacity $\bar{W}$ with $M$ for different values of $\bar{h}_{m}$ with $\beta=0.05, \bar{l}=0.3$, $\bar{c}=0.3$, and $\psi=0.01$.

physical reason being that larger values of permeability lead to more voids available on the porous escape of the lubricant. Thus, the porous facing becomes the main path for lubricant flow and therefore the modified film thickness due to the presence of surface asperity has a negligible effect. However, this decrease in $\bar{W}$ due to $\psi$ can be compensated by the

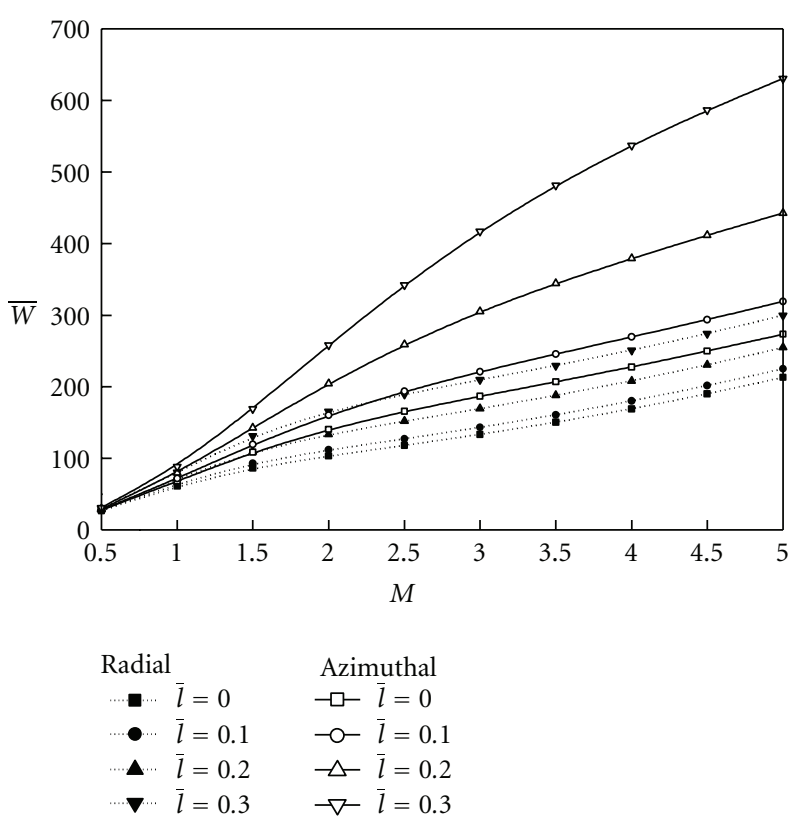

FIGURE 7: Variation of nondimensional mean load-carrying capacity $\bar{W}$ with $M$ for different values of $\bar{l}$ with $\beta=0.05, \bar{c}=0.3$, and $\psi=0.01$

appropriate choice of lubricants with additives and also by the appropriate choice of roughness pattern on the bearing surfaces.

4.3. MHD Squeeze-Film Time. The response time of the squeeze film is one of the significant factors in the design of bearings. The response time is the time that will elapse for a squeeze film to be reduced to some minimum permeability height. Figure 10 displays the variation of nondimensional response time $\bar{t}$ as a function of the sphere displacement $1-\bar{h}_{m}$ for different values of magnetization number $M$.

The initial condition $\bar{h}_{m}(0)=1$ is used for the squeezing motion of the system. Therefore the initial displacement is $1-\bar{h}_{m}(0)=0$. At this displacement, the effect of applied magnetic field is observed to increase the value of response time and this increase is more pronounced in either case as compared to the non-conducting lubricant case.

The variation of $\bar{t}$ with $1-\bar{h}_{m}$ for different values of couple stress parameter, $\bar{l}$, is plotted in Figure 11. It is found that due to the presence of effects of couple stresses, the squeeze-film time increases/decreases for the azimuthal/radial roughness pattern as compared to the corresponding Newtonian case $(\bar{l}=0.0)$.

Figure 12 shows the effect of roughness parameter $\bar{c}$ on the variation of $\bar{t}$ with $1-\bar{h}_{m}$ for both types of roughness patterns. It is interesting to note that the effect of $\bar{c}$ is to increase/decrease the response time of the squeeze film for the azimuthal/radial roughness pattern as compared to the corresponding smooth case $(\bar{c}=0.0)$. The effect of porous parameter $\psi$ on the variation of $\bar{t}$ with $1-\bar{h}_{m}$ is shown in Figure 13. It is observed that the response time reduces 


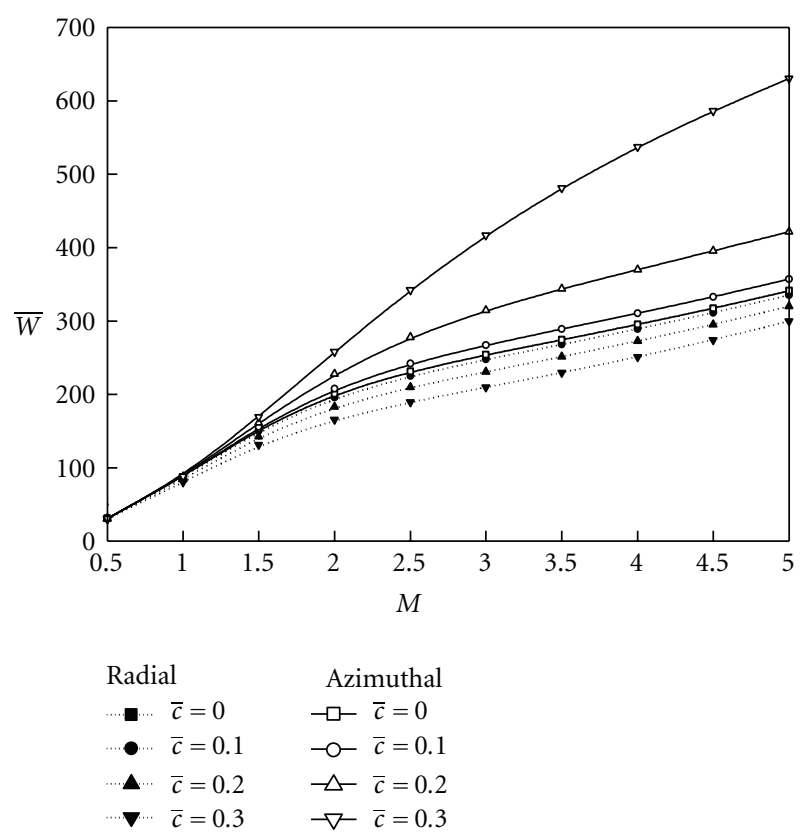

FIGURE 8: Variation of nondimensional mean load-carrying capacity $\bar{W}$ with $M$ for different values of $\bar{c}$ with $\beta=0.05, \bar{l}=0.3$, and $\psi=0.01$.

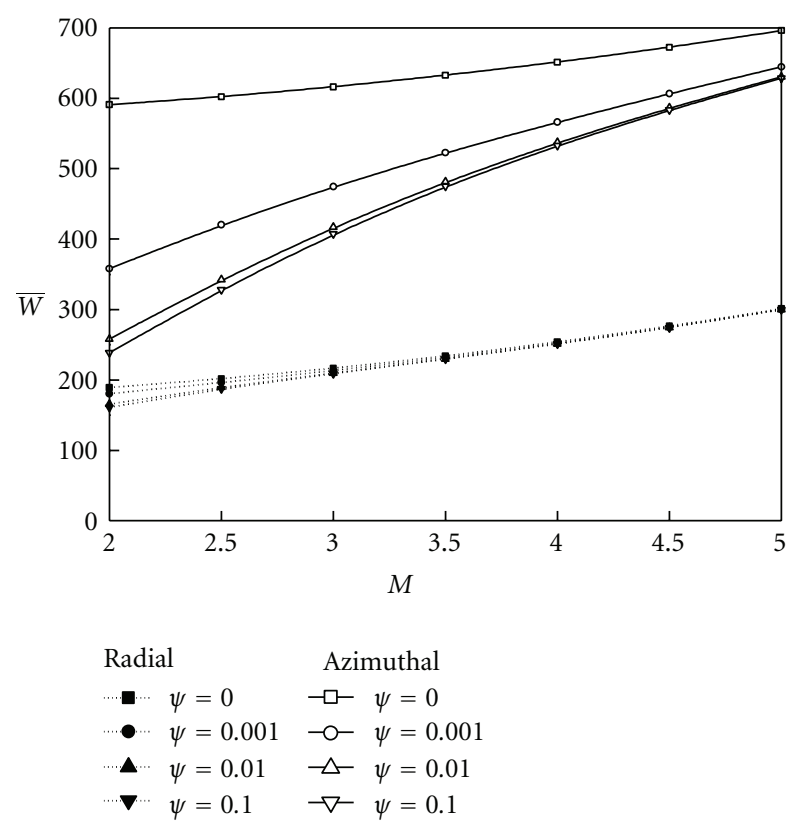

FIGURE 9: Variation of nondimensional mean load-carrying capacity $\bar{W}$ with $M$ for different values of $\psi$ with $\beta=0.05, \bar{l}=0.3$ and $\bar{c}=0.3$.

for the increasing values of $\psi$ for both types of roughness patterns. As the sphere moves downwards, the fluid is forced into the pores of the porous material and hence resulting in the lapse of the response time that is, the time of approach of a sphere to the plane surface is delayed.

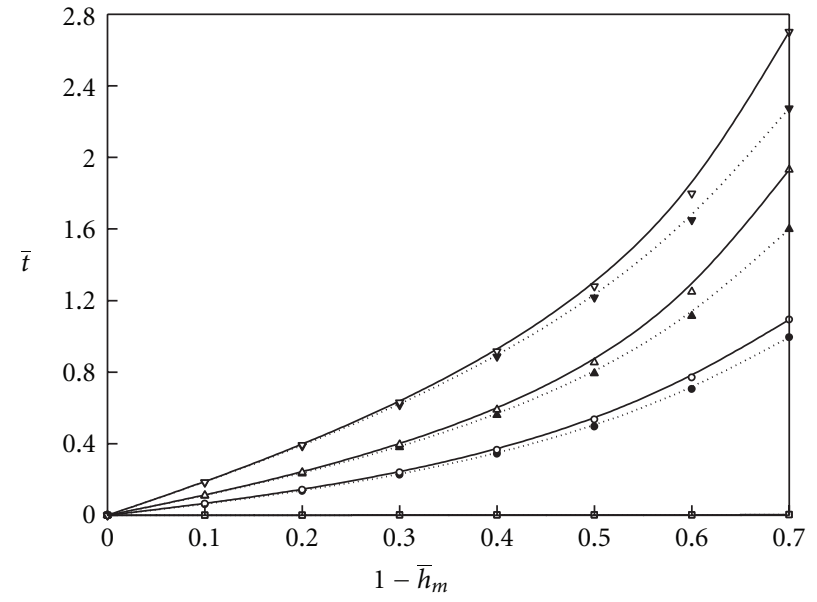

$$
\begin{aligned}
& \text { Radial Azimuthal } \\
& \text {-... } M=0 \quad \square-M=0 \\
& \text {-... } M=1 \quad-0-M=1 \\
& \triangle M=2 \quad-M=2 \\
& \text { - } \cdots=3 \quad-\nabla-M=3
\end{aligned}
$$

FIGURE 10: Variation of nondimensional response time $\bar{t}$ with $1-\bar{h}_{m}$ for different values of $M$ with $\beta=0.05, \bar{l}=0.3, \bar{c}=0.3$, and $\psi=$ 0.01 .

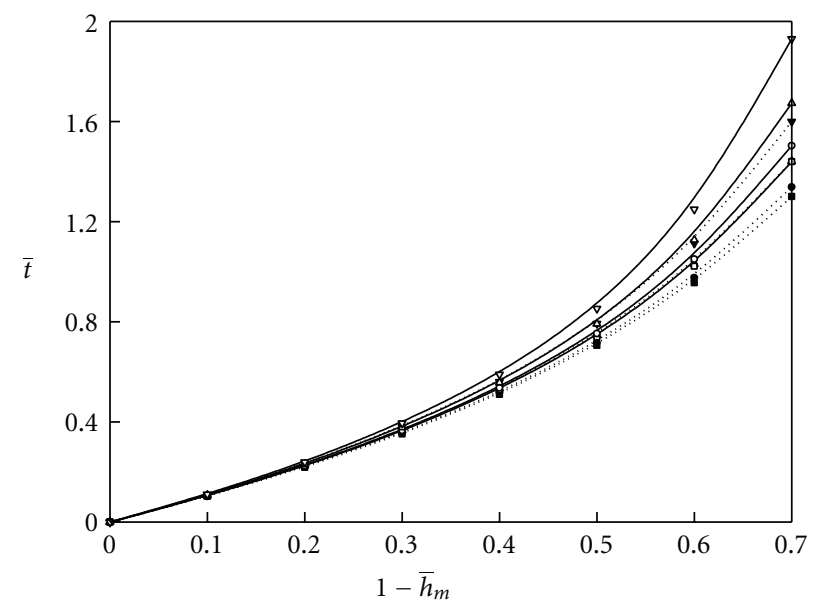

$$
\begin{aligned}
& \text { Radial Azimuthal } \\
& \text {..... } \bar{l}=0 \quad-\square-\bar{l}=0 \\
& \text { - } \bar{l}=0.1 \quad-0-\bar{l}=0.1 \\
& \Delta \cdots \bar{l}=0.2 \quad \triangle \quad \bar{l}=0.2 \\
& \boldsymbol{\nabla} \bar{l}=0.3 \quad \rightarrow-\bar{l}=0.3
\end{aligned}
$$

FIGURE 11: Variation of nondimensional response time $\bar{t}$ with $1-\bar{h}_{m}$ for different values of $\bar{l}$ with $\beta=0.05, M=2, \bar{c}=0.3$, and $\psi=$ 0.01 .

\section{Design Example}

The following mathematical demonstration of MHD couple stress squeeze-film characteristics between a sphere and a rough porous plane surface is considered, for the illustration of engineering design application. By using the values of 


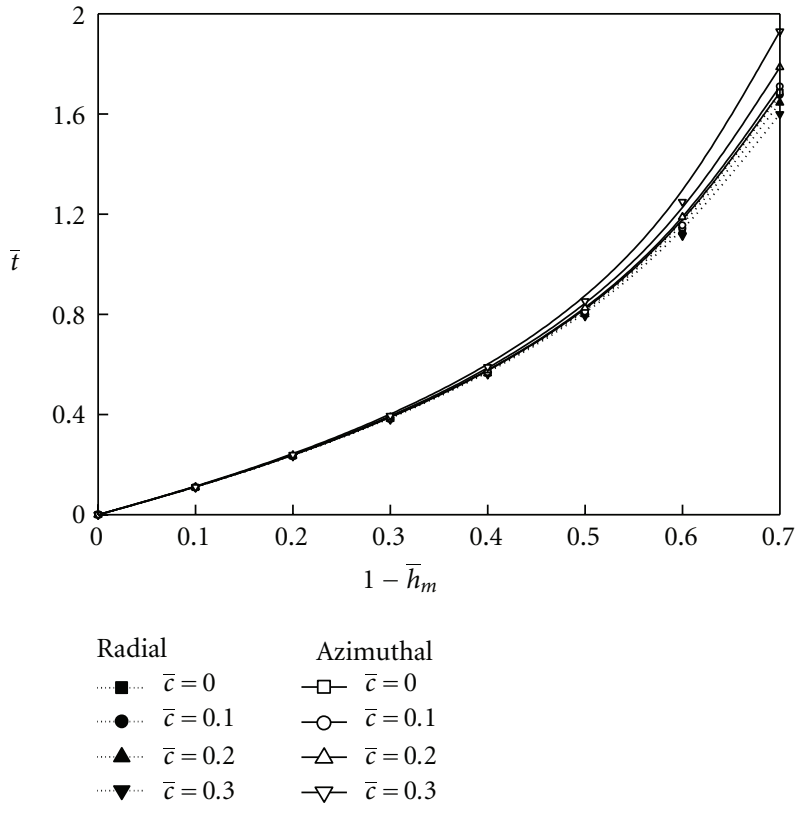

FIGURE 12: Variation of nondimensional response time $\bar{t}$ with $1-\bar{h}_{m}$ for different values of $\bar{c}$ with $\beta=0.05, M=2, \bar{l}=0.3$, and $\psi=0.01$.

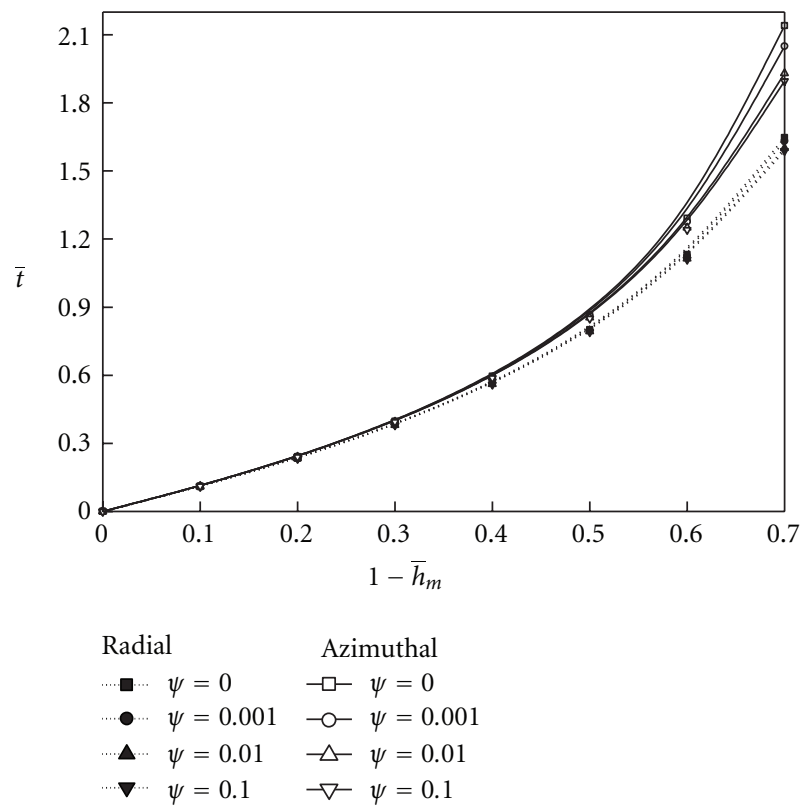

FIGURE 13: Variation of nondimensional response time $\bar{t}$ with $1-\bar{h}_{m}$ for different values of $\psi$ with $\beta=0.05, M=2, \bar{l}=0.3$, and $\bar{c}=0.3$.

various dimensional parameters, one can obtain a design example for the engineering design application as follows:

$$
\begin{aligned}
& R=2 \mathrm{~mm}, \mu=500 \mathrm{cp}, h_{m_{0}}=0.1 \mathrm{~mm}, \\
& \sigma=1.07 \times 10^{6} \mathrm{mh}_{0} / \mathrm{m} \\
& H_{0}=0.8 \mathrm{~mm}, h_{m}=0.01 \mathrm{~mm}-0.09 \mathrm{~mm} \\
& k=1.25 \times 10^{-12} \mathrm{~m}^{2}, 1.25 \times 10^{-11} \mathrm{~m}^{2}, 1.25 \times 10^{-10} \mathrm{~m}^{2}
\end{aligned}
$$

$$
\begin{aligned}
& c=0.01 \mathrm{~mm}, 0.02 \mathrm{~mm}, 0.03 \mathrm{~mm} \\
& B_{0}=0.216 \mathrm{~Wb} / \mathrm{m}^{2}, 0.432 \mathrm{~Wb} / \mathrm{m}^{2}, 0.648 \mathrm{~Wb} / \mathrm{m}^{2} .
\end{aligned}
$$

With the help of definitions of various nondimensional parameters, one can obtain

$$
\begin{aligned}
M & =0.0,1.0,2.0,3.0, \quad \bar{l}=0.0,0.1,0.2,0.3, \\
\bar{c} & =0.0,0.1,0.2,0.3, \\
\psi & =0.001,0.01,0.1, \quad h_{m}=0.01-0.9 .
\end{aligned}
$$

The equations (27) and (29) give the MHD couple stress load-carrying capacity and MHD couple stress squeeze-film time and are displayed in the Figures 2-13. Now, with the help of this design example, the design engineers can design the most advantageous MHD couple stress squeezefilm behaviour between a sphere and a rough porous plane surface.

\section{Conclusion}

The effect of surface roughness on the MHD squeeze-film characteristics between a sphere and a porous plane surface is presented on the basis of Christensen stochastic theory for rough surfaces. On the basis of results discussed above, the following conclusions can be drawn.

(1) The presence of an externally applied transverse magnetic field provides an enhancement in the loadcarrying capacity and response time as compared to the non-conducting case for both types of roughness patterns.

(2) The couple stress effects are more pronounced for azimuthal roughness pattern as compared to the radial roughness pattern. Due to the presence of additives in the fluid, the significant increase in loadcarrying capacity and squeeze-film time is observed than the Newtonian case for both types of roughness patterns.

(3) The roughness of the surface causes a reasonable effect on the characteristics of the bearing. As the surface asperity increases, the large amount of load is delivered in the bearing and lengthens the response time of squeeze-film motion as compared to the smooth case. The effect of surface roughness is more accentuated in the case of azimuthal roughness pattern than that of radial roughness pattern.

(4) The permeability of the porous layer diminishes the squeeze-film characteristics as compared to the nonporous case. The raise in the height of the porous layer motivates the fall in the squeeze-film pressure and the load-carrying capacity and thus shortens the response time and also the possibility of sphere-toplane contact. 


\section{Nomenclature}

$B_{0}: \quad$ Applied magnetic field

c: $\quad$ Roughness parameter

$\bar{c}$ : $\quad$ Nondimensional roughness parameter $\left(c / h_{m_{0}}\right)$

$h$ : $\quad$ Film thickness $\left(=h_{m}+\left(r^{2} / 2 R\right)\right)$

$h_{m}$ : Minimum film thickness

$h_{m_{0}}$ : Initial minimum film thickness

$\bar{h}: \quad$ Nondimensional film thickness $\left(=h / h_{m_{0}}\right)$

$\bar{h}_{m}$ : Nondimensional minimum film thickness $\left(=h_{m} / h_{m_{0}}\right)$

$h_{s}: \quad$ Stochastic film thickness

$H_{0}$ : Thickness of porous facing

$k$ : $\quad$ Permeability of porous facing

$m$ : Porosity

M: $\quad$ Magnetic Hartmann number

$\bar{p}: \quad$ Nondimensional film pressure

$R: \quad$ Radius of the sphere

$r, z$ : Radial and axial coordinates

$u, w$ : Velocity components of the fluid in the film region

$u^{*}, w^{*}$ : Velocity components of the fluid in the porous region

$W: \quad$ Load-carrying capacity

$\bar{W}: \quad$ Non-dimensional load-carrying capacity

$t: \quad$ Time

$\bar{t}$ : Nondimensional response time.

\section{Greek Symbols}

$\beta$ : Nondimensional central film thickness

$\sigma$ : Fluid conductivity

$\bar{\sigma}$ : Standard deviation

$\mu$ : Fluid viscosity

$\psi$ : Porous parameter $\left(=k H_{0} / h_{m_{0}}^{3}\right)$

$\xi:$ Random variable.

\section{Acknowledgment}

The authors are thankful to Dr. N. B. Naduvinamani, Professor, Department of Mathematics, Gulbarga University, Gulbarga for his valuable discussions.

\section{References}

[1] T. Ariman, M. A. Turk, and N. D. Sylvester, "Microcontinuum fluid mechanics—a review," International Journal of Engineering Science, vol. 11, no. 8, pp. 905-930, 1973.

[2] T. Ariman, M. A. Turk, and N. D. Sylvester, "Applications of microcontinuum fluid mechanics," International Journal of Engineering Science, vol. 12, no. 4, pp. 273-293, 1974.

[3] V. K. Stokes, "Couple stresses in fluids," Physics of Fluids, vol. 9, no. 9, pp. 1709-1715, 1966.

[4] G. Ramanaiah, "Squeeze films between finite plates lubricated by fluids with couple stress," Wear, vol. 54, no. 2, pp. 315-320, 1979.

[5] W. F. Hughes and R. A. Elco, "MHD lubrication flow between parallel rotating disks," Journal of Fluid Mechanics, vol. 13, pp. 21-32, 1962.
[6] D. C. Kuzma, E. R. Maki, and R. J. Donnely, "The MHD squeeze film," Journal of Fluid Mechanics, vol. 19, pp. 395-400, 1964.

[7] P. Gould, "High-pressure spherical squeeze films," Journal of Lubrication Technology, vol. 93, no. 1, pp. 207-208, 1971.

[8] H. D. Conway and H. C. Lee, "Impact of a lubricated surface by a sphere," Journal of Lubrication Technology, vol. 97, no. 4, pp. 613-615, 1975.

[9] E. A. Hamza, "Magnetohydrodynamic squeeze film," Journal of Tribology, vol. 110, no. 2, pp. 375-377, 1988.

[10] J. R. Lin, "Squeeze film characteristics between a sphere and a flat plate: couple stress fluid model," Computers and Structures, vol. 75, no. 1, pp. 73-80, 2000.

[11] T. G. Cowling, "Magneto-hydrodynamics," Interscience, 1957.

[12] I. Horia and C. R. Ene, Academy of Sciences at Paris A and B, vol. 268, p. 564, 1969.

[13] P. D. S. Verma, "Magnetic fluid-based squeeze film," International Journal of Engineering Science, vol. 24, no. 3, pp. 395401, 1986.

[14] M. V. Bhat and G. M. Deheri, "Magnetic fluid based squeeze film between porous circular disks," Journal of Indian Academy of Mathematics, vol. 15, pp. 145-147, 1993.

[15] J. Prakash and S. Vij, "Load capacity and time-height relations for squeeze films between porous plates," WEAR, vol. 24, no. 3, pp. 309-322, 1973.

[16] R. C. Shah and M. V. Bhat, "Squeeze film based on magnetic fluid in curved porous rotating circular plates," Journal of Magnetism and Magnetic Materials, vol. 208, no. 1-2, pp. 115119, 2000.

[17] H. Christensen, "Stochastic model for hydrodynamic lubrication of rough surfaces," Proceedings of the Institution of Mechanical Engineers, vol. 184, pp. 1013-1026, 1970.

[18] H. G. Elrod, "Thin-film lubrication theory for newtonian fluids with surfaces possessing striated roughness or grooving," Journal of Lubrication Technology, vol. 95, no. 4, p. 484, 1973.

[19] J. Prakash and K. Tiwari, "Lubrication of a porous bearing with surface corrugations," Journal of Lubrication Technology, vol. 104, no. 1, pp. 127-134, 1982.

[20] J. Prakash and K. Tiwari, "Effect of surface roughness on the squeeze film between rotating porous annular discs," Journal of Mechanical Engineering Science, vol. 24, no. 3, pp. 155-161, 1982.

[21] K. Gururajan and J. Prakash, "Effect of surface roughness in a narrow porous journal bearing," Journal of Tribology, vol. 122, no. 2, pp. 472-475, 2000.

[22] N. B. Naduvinamani, P. S. Hiremath, and G. Gurubasavaraj, "Surface roughness effects in a short porous journal bearing with a couple stress fluid," Fluid Dynamics Research, vol. 31, no. 5-6, pp. 333-354, 2002.

[23] N. M. Bujurke and R. B. Kudenatti, "MHD lubrication flow between rough rectangular plates," Fluid Dynamics Research, vol. 39, no. 4, pp. 334-345, 2007.

[24] T. L. Chou, J. W. Lai, and J. R. Lin, "Magneto-hydrodynamic squeeze film characteristics between a sphere and a plane surface," Journal of Marine Science and Technology, vol. 11, no. 3, pp. 174-178, 2003.

[25] N. B. Naduvinamani and M. Rajashekar, "MHD Couplestress squeeze-film characteristics between a sphere and a plane surface," Tribology-Materials, Surfaces \& Interfaces, vol. 5, pp. 94-99, 2011. 

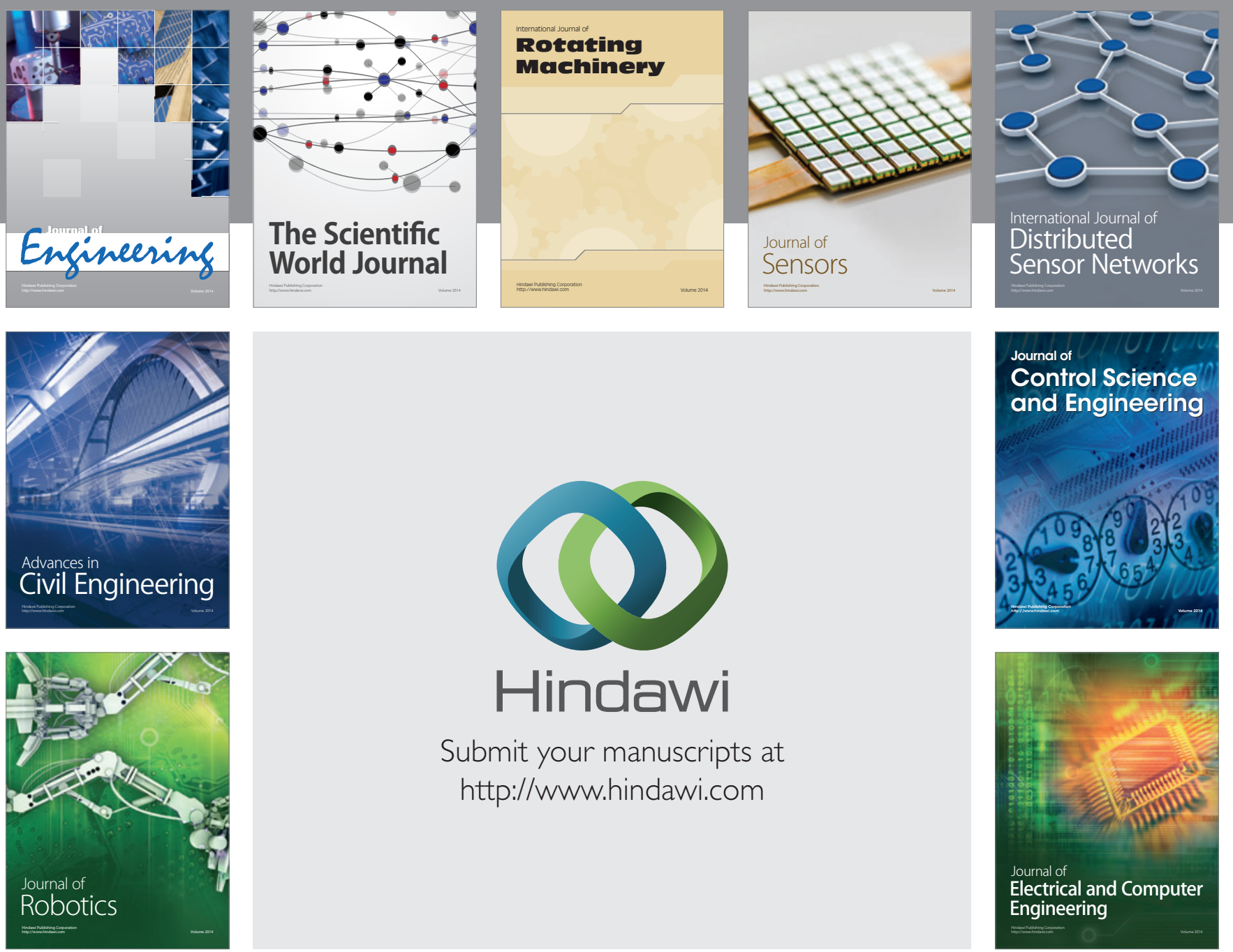

Submit your manuscripts at

http://www.hindawi.com
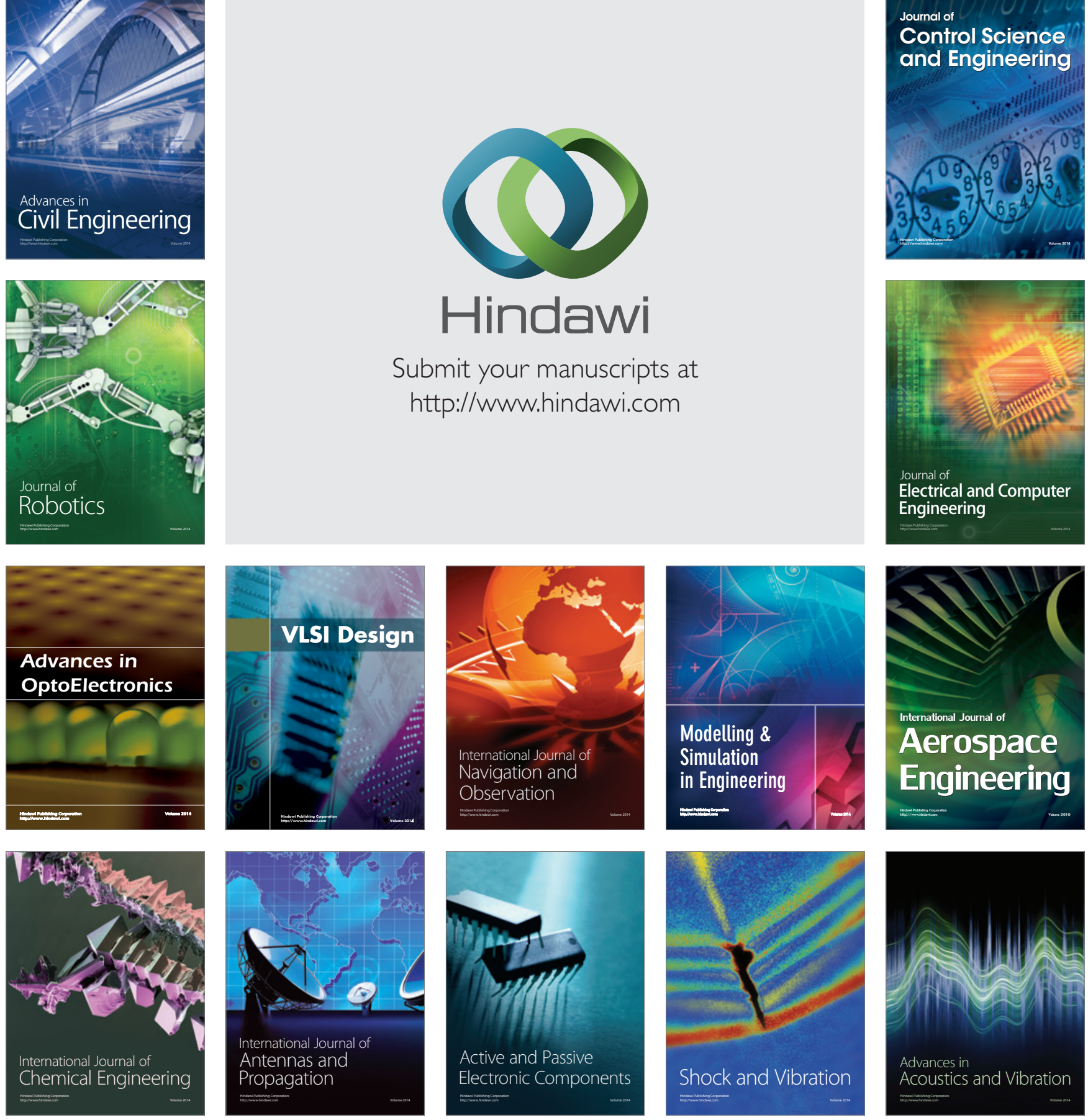